Assessment of Risk Factors History of ankle sprain in the past year was defined in a time-dynamic manner as any bilateral ankle sprain (game or non-game) in the 365 days prior to the game of interest. Hazard ratios (HR) and $95 \%$ confidence intervals (CI) were calculated while controlling for years in the NBA, body mass index, and average NBA minutes played in the prior year.

Main Outcome Measurements All acute-onset ankle sprains reported in games were identified from the NBA standardized electronic medical record ( $\mathrm{n}=30$ teams).

Results Across this 4-season study, 482 incident ankle sprains were reported in NBA games among 681 players and $2,517,549$ player-minutes. Of the players that sustained an incident sprain, 44\% $(n=211)$ had at least one ankle sprain in the prior year. Compared to players with no sprains in the past year, the risk of incident ankle sprain increased with increasing number of prior ankle sprains; a $28 \%$ increase in risk $(\operatorname{adjHR}=1.28,95 \%$ CI $1.03,1.58)$ with one prior sprain, a $51 \%$ increase in risk $(\operatorname{adjHR}=1.51,95 \%$ CI 1.10, 2.04) with two prior sprains, and a $100 \%$ increase in risk (adjHR $=2.00,95 \%$ CI $1.31,2.94)$ with three or more prior sprains.

Conclusions History of ankle sprain in the past year was associated with increased risk of incident ankle sprain among NBA players. $44 \%$ of players had at least one ankle sprain (game or non-game) within one year prior to the incident sprain.

\section{AN UNSUPERVISED E-HEALTH SUPPORTED NEUROMUSCULAR TRAINING PROGRAM IS NOT EFFECTIVE IN THE PREVENTION OF RECURRENT ANKLE SPRAINS IN PATIENTS IN PRIMARY CARE: THE TRAPP- STUDY}

${ }^{1}$ Adinda Mailuhu, ${ }^{2}$ Evert Verhagen, ${ }^{1} J o h n$ Van Ochten, ${ }^{1}$ Patrick Bindels, 'Sita BiermaZeinstra, ${ }^{1}$ Marienke Van Middelkoop. 'Department of General Practice, Erasmus MC, University Medical Centre, Rotterdam, Netherlands; ${ }^{2}$ Department of Health Sciences and EMGO Institute for Health and Care Research, VU University Medical Center, Amsterdam, Netherlands

\subsection{6/bjsports-2021-IOC.102}

Background Ankle sprains are one of the most frequent injuries of the musculoskeletal system. No optimal treatment strategy has proven to be effective in general practice, however promising results were achieved in a preventive trial among athletes

Objective To examine the effectiveness of an unsupervised ehealth supported neuromuscular training program in combination with usual care in general practice compared to usual care alone in patients with acute lateral ankle sprains in general practice

Design Randomised Controlled Trial

Setting Primary care

Patients (or Participants) Patients (14-65 years) who visited a general practitioner with an acute lateral ankle sprain within three weeks of injury

Interventions (or Assessment of Risk Factors) The intervention group received, in addition to usual care, an unsupervised ehealth supported neuromuscular training program, and the control group received usual care alone

Main Outcome Measurements The primary outcome was selfreported recurrent sprains during follow-up. Secondary outcomes were ankle function (Ankle Function Score 0-100), pain in rest and during activity (numerical rating scale $0-10$ ), subjective recovery and return to the same type and level of sport.

Results 165 participants were included with a mean age of 38.3 (SD 14.2) years and $41.8 \%$ were male. No difference in the occurrence of a recurrent sprain was found between the intervention $(\mathrm{n}=17 ; 20.7 \%)$ and control group $(\mathrm{n}=20$; 24.1\%) (HR 1.14; 95\% CI 0.59-2.21). Also, no differences in secondary outcomes were found between groups. The compliance rate with the program was low (6.1\%).

Conclusions Although the recurrence rate of ankle sprains was relatively high, an unsupervised e-health supported neuromuscular training program is not effective in preventing recurrent sprains in patients with an acute lateral ankle sprain in general practice. More research is necessary to indicate the best treatment modality for this group of patients.

\section{\begin{tabular}{|l|l}
110 & UNDERSTANDING THE IMPACT OF ANKLE INJURIES
\end{tabular} AMONG CONTEMPORARY PRE-PROFESSIONAL DANCERS: INCIDENCE AND RISK FACTORS}

${ }^{1}$ Adinda Mailuhu, ${ }^{2}$ Rogier van Rijn, 1,2,3,4 Janine Stubbe, ${ }^{1}$ Sita MA Bierma-Zeinstra, ${ }^{1}$ Marienke van Middelkoop. 'Department of General Practice, Erasmus MC University Medical Center, Rotterdam, Netherlands; ${ }^{2}$ Codarts, University of the Arts, Rotterdam, Netherlands; ${ }^{3}$ PErforming artist and Athlete Research Lab (PEARL), Rotterdam, Netherlands: ${ }^{4}$ Rotterdam Arts and Sciences Lab (RASL), Rotterdam, Netherlands

\subsection{6/bjsports-2021-IOC.103}

Background Ankle injuries are common injuries among preprofessional dancers. An overview of the incidence and risk factors is however lacking.

Objective To examine the incidence and risk factors of ankle injuries among contemporary pre-professional dancers.

Design Prospective cohort study.

Setting Codarts University of the Arts, Rotterdam, The Netherlands.

Patients (or Participants) 91 first-year contemporary pre-professional dancers, from Bachelor Dance $(n=59)$ and Bachelor Dance Teacher $(n=32)$ were included.

Interventions (or Assessment of Risk Factors) At the start of the academic year all dancers completed a questionnaire, underwent a physical screening measuring the ankle range of motion (ROM) and dorsiflexion. During the academic year, all dancers completed monthly questionnaires on the occurrence of an ankle injury that were referred to in the Oslo Sports Trauma Research Center (OSTRC) questionnaire. Injuries were defined into substantial injuries (score $\geq 13$ on question 2 or 3 of OSTRC questionnaire), injuries leading to medical attention and to dance time-loss. Potential risk factors for an ankle injury included dancer characteristics, history of ankle injury in previous year, ankle ROM and dorsiflexion.

Main Outcome Measurements The number of self-reported ankle injuries during one academic year.

Results 17 (18.7\%) dancers reported an ankle injury, with a total of 33 injuries. Of these dancers reporting an ankle injury, $82.4 \%$ led to dance time-loss (mean number of 19.9 days unable to fully participate), $47.1 \%$ to medical attention and $41.2 \%$ were substantial injuries. No significant risk factors for ankle injuries could be identified. 